\title{
Evaluating the Benefit of a Smart Scheduler in a Non-Cooperative, Multi-User Heterogeneous Wireless ITS Environment
}

\author{
Cristian Roman, Peter Ball, Shumao Ou \\ School of Engineering, Computing and Mathematics, Oxford Brookes University, Oxford, UK \\ Email: \{cristian.roman, pball, sou\}@brookes.ac.uk
}

\begin{abstract}
Heterogeneous wireless networks will play a significant role in providing multiservice connectivity in ITS, and in particular vehicular networks. This paper describes a smart scheduling approach that allows end user nodes to direct packets over the best available wireless access technologies and set priorities for selected services. The performance of this smart scheduler has been simulated in a non-cooperative multi user environment and the results show that, for the prioritised services, the scheduler can provide a lower average packet delay and a higher average packet delivery ratio for all users than a wireless system that selects on signal strength alone.
\end{abstract}

\section{INTRODUCTION}

Intelligent Transport Systems (ITS) need to be able to provide reliable broadband access to users in a variety of transport modes. The goal is to meet the Always-Best-Connected (ABC) paradigm [1]. Developments in cellular networks such as LTE and the future introduction of $5 \mathrm{G}$ cellular services will increase the bandwidth available for mobile users, but in order to achieve cost effective and widespread coverage, heterogeneous wireless solutions are likely to play a significant role.

There are two approaches to provide heterogeneity to nodes in ITS. The first adopts a centralised approach where a network operator controls the different Radio Access Technologies (RATs). The operator can thus specify the RATs that each node should use. In many situations, available RATs are not controlled by the same provider, in which case the provider can not manage the transfer of data between the different RATs. The second approach is for the selection to take place at the user node, independently of the RAT provider. The advantage of this approach is that the user is able to observe and possibly use RATs from different operators but also use technologies which are not centrally controlled. Most current non-centralised systems adopt a non-cooperative approach (e.g. Wi-Fi) where each user seeks to maximise their usage, without consideration of other users.

The second approach enables mobile nodes to select the most suitable radio access technology (RAT) that is currently available in their location. In addition, it may also be beneficial to have packets sent simultaneously over multiple RATs to increase the available bandwidth. To support Quality-ofService (QoS) requirements it is important to prioritise certain packets; for example to meet the reliability requirements for safety critical services and, the delay requirements for real time services. The node therefore requires a smart scheduling algorithm that is able to queue and switch packets to the most suitable RAT.

Work has been reported which shows that a heterogeneous wireless approach with a smart scheduling algorithm can deliver improved performance for a single user [2] [3]. This paper extends the analysis to investigate the benefit of a heterogeneous wireless smart scheduling algorithm solution to a multi-user non-cooperative network. The approach taken in this paper is to demonstrate the utility and feasibility of such a smart algorithm within a vehicular network and to demonstrate packet prioritisation which enables stringent safety requirements to met. This approach can be applied to any mobile node in ITS. The results show that by having heterogeneous access to multiple networks combined with a smart scheduler, the performance of the prioritised packets can be enhanced for all users.

The rest of the article is organised as follows. First, Section II reviews related work on multi-user heterogeneous wireless access. Section III describes the smart packet scheduler. Section IV describes the system and the simulation environment used to evaluate the performance. Section $\mathrm{V}$ presents the results. Section VI discusses further work. Finally, Section VII presents the conclusions.

\section{RELATED WORK}

Since the infrastructure of cellular networks is already deployed, it is economically efficient to utilise cellular networks to support V2I communications [4]. Hossain et al. [5] state that an Advanced Heterogeneous Vehicular Network (AHVN) that uses multiple radios and multiple access technologies in a collaborative manner could be the best candidate for a vehicular network. Their key motivation is that the Dedicated Short Range Communications (DSRC) technology will only be effective when it is widely available, governments legislate for DSRC deployment in passenger vehicles, and older noncompliant vehicles have been retired. 
Lequerica et al. [6] have shown that cellular system-aided (3G/LTE) heterogeneous vehicular networks can greatly facilitate message dissemination in terms of message delivery ratio, outperforming pure vehicular ad-hoc networks with sparsely placed vehicles. Based on this research Ho Ting Chen et al. [7] state that one viable option to improve the network connectivity in VANETs for infotainment and road safety service support is via the assistance of a well-established cellular system as a complementary network. However, not all generations of cellular technologies are adequate for use in Vehicle to Infrastructure (V2I) communications. The 3G system (WCDMA/UMTS) does not provide good support for safety services in vehicular communications. For instance, the connection setup from an idle state requires 2 to $2.5 \mathrm{~s}$. The delivery latency in all the states is larger than the allowed maximum latency for safety services $(100 \mathrm{~ms})$.

LTE (4G) is envisioned to support V2I communications especially in the initial deployment stage of vehicular networks and play an important role in rural areas where the vehicle density is low. LTE meets most of the application QoS requirements in terms of reliability, scalability, and mobility support; however, it may not be able to meet the stringent delay requirements of safety critical services when the cellular traffic load is high [8]. This is partly due to the fact that in current LTE systems, the MAC layer lacks an efficient scheduling mechanism for mapping of vehicular traffic features to the existing QoS Class Identifier (QCI) [9]. In addition, in highly dense areas, such as cities with numerous building reflections, the performance of cellular connectivity, for both LTE and WiMax, varies based on the number of users [10].

Until 5G or a new technology is developed for ITS, and the corresponding infrastructure deployed, an alternative option is to combine the current available wireless technologies in a multi-RAT bundle to represent all the opportunities in the plurality in access. The case of integration between RATs is studied by Karimi et al. [11] where it is shown that the gain of heterogeneous networks, over independent RATs, in terms of throughput and spectral efficiency varies between $15 \%$ and $60 \%$ [12]. A summary table, similar to that of Jiau et al. [13], depicting the possible RATs to be used in ITS systems can be found in Table I and an illustration of the possible network connections in ITS is shown in Fig. 1.

One of the complementary networks that could be used to support an integrated approach, and offload some of the traffic from cellular connections, is the existing Wi-Fi technology with Access Points (APs) distributed around a city [14]. Popular standards available on mobile devices (laptops, phones) include the $802.11 \mathrm{~b}, \mathrm{~g}, \mathrm{n}$ and, more recently, higher data rates are reached with $802.11 \mathrm{ac}$. To solve packet priority issues, a QoS approach has been taken by the 802.11e amendment with a traffic type classification mechanism. These standards provide good connectivity for nodes that have limited mobility and that do not require extensive handover services.

Even though Wi-Fi suffers from high network setup time [15], 802.11n without any modification can still be useful in congested city areas where node speed is low. Bychkovsky et

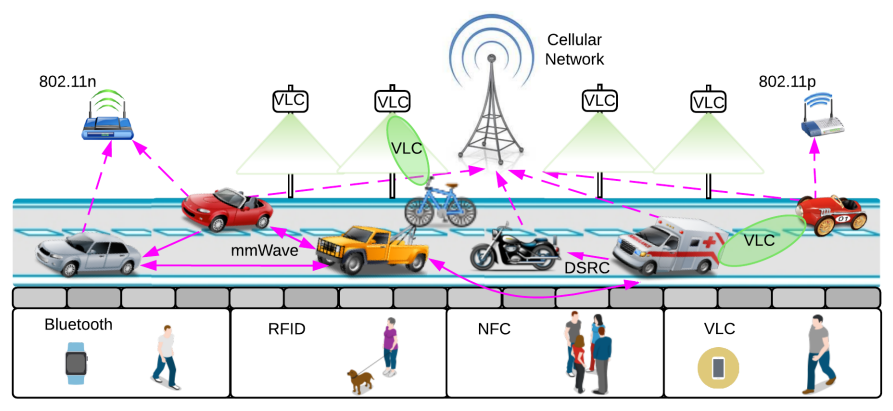

Fig. 1. Architecture of Wireless Heterogeneous ITS Network

al. [16] revealed that in urban environments, after a vehicle associates with an AP and acquires an IP address, connection time ranges from 5 to 24 seconds. Standard Wi-Fi can provide an alternative for non-safety messages and certain safety messages (e.g. traffic hazard warning). This can release the cellular network from extra pressure and reduce the cost in the initial stage of deployment. The state of the art progress, as well as future research directions and challenges, for Wi-Fi offloading is surveyed by He et al. [14].

The de-facto standard for Vehicle-to-Everything (V2X) communication is DSRC, which is based on the IEEE 802.11p standard. However, work by Han et al. [17] expresses concerns that DSRC can exhibit poor performance in the event of a large number of vehicles. Heterogeneous networks would be a natural candidate but Bazzi [18] has shown that, although the use of technology combining allows higher throughput for a single user and helps reduce packet losses, leading to improved efficiency and throughput without consuming much wireless bandwidth [19], it maybe a counter-productive strategy in a scenario with multiple users acting selfishly [18] [20]. This paper investigates whether it might be possible to get improved performance in a multi-user non cooperative environment through the use of a priority scheduling scheme, delivering packets based on the surrounding context (e.g. network conditions).

Aggregating multiple network technologies can occur at different layers of the OSI layered architecture. A summary and comprehensive list of some of the heterogeneous aggregation solutions, can be found in [21] or [22]. As an example, in previously reported work, scheduling without modification of the wireless standards has been carried out at the application layer [23], transport layer [18] and network layer [24]. The advantages and disadvantages of these approach have been previously analysed in [3].

\section{Description OF APPROACH - ShIM LAYER}

Each communication standard has unique features at the physical and MAC layers. Above these layers, many communication systems adopt the TCP/IP layered architecture. To implement a flexible heterogeneous network which retains the properties of the data source but can adapt to the selected wireless access technology, a shim layer, located between the 
TABLE I

COMPARISON OF WIRELESS COMMUNICATIONS FOR VEHICULAR NETWORKS

\begin{tabular}{|c|c|c|c|c|c|c|c|c|c|}
\hline & Short Radio Wi-Fi & & Cellular & & & & Other & & \\
\hline & Wi-Fi (WLAN) & DSRC/WAVE & $3 G$ & WiMAX & $4 \mathrm{G}$ & $4 \mathrm{G}$ & VLC & Bluetooth & mmWave \\
\hline & $802.11 \mathrm{a} / \mathrm{b} / \mathrm{g} / \mathrm{n}$ & $802.11 p$ & UMTS & 802.16 & LTE & LTE-A & 802.15 .7 & 802.15 .1 & $802.15 .3 \mathrm{c}$ \\
\hline Frequency & $\begin{array}{r}{[2.401-2.495} \\
5.15-5.725 \mathrm{GHz}]\end{array}$ & $\begin{array}{r}{[5.850-} \\
5.925 \mathrm{GHz}]\end{array}$ & $\begin{array}{r}\text { [700- } \\
2600 \mathrm{MHz}]\end{array}$ & $\begin{array}{r}{[2.5-} \\
2.69 \mathrm{GHz}]\end{array}$ & $\begin{array}{r}{[700-} \\
2600 \mathrm{MHz}]\end{array}$ & $\begin{array}{r}{[450 \mathrm{MHz}} \\
-4.99 \mathrm{GHz}]\end{array}$ & $\begin{array}{r}{[400-} \\
800 \mathrm{THz}]\end{array}$ & $\begin{array}{r}{[2.4-} \\
2.485 \mathrm{GHz}]\end{array}$ & $\begin{array}{r}{[57-} \\
66 \mathrm{GHz}]\end{array}$ \\
\hline $\begin{array}{l}\text { Channel } \\
\text { Width }\end{array}$ & $\begin{array}{r}10,20,40 \\
80,160 \mathrm{MHz}\end{array}$ & $\begin{array}{r}5,10,20 \\
\mathrm{MHz}\end{array}$ & $5 \mathrm{MHz}$ & $\begin{array}{r}1.25,5, \\
10,20 \mathrm{MHz}\end{array}$ & $\begin{array}{l}1.4,3,5,10, \\
15,20 \mathrm{MHz}\end{array}$ & $100 \mathrm{MHz}$ & $\begin{array}{r}1 \\
\text { Pixel }\end{array}$ & $1 \mathrm{MHz}$ & $2.16 \mathrm{GHz}$ \\
\hline Bit Rate & $\begin{array}{r}\text { Medium }(\mathrm{a} / \mathrm{b} / \mathrm{g}) \\
2.7-54 \mathrm{Mbps}\end{array}$ & $\begin{array}{r}\text { Medium } \\
\text { 3-27 Mbps }\end{array}$ & $\begin{array}{r}\text { Low } \\
2 \mathrm{Mbps}\end{array}$ & $\begin{array}{r}\text { High } \\
70 \mathrm{Mbps}\end{array}$ & $\begin{array}{l}\text { Very High } \\
300 \mathrm{Mbps}\end{array}$ & $\begin{array}{r}\text { Very High } \\
1 \text { Gbps }\end{array}$ & $\begin{array}{r}\text { Low } \\
10 \mathrm{Mbps}\end{array}$ & $\begin{array}{r}\text { Low } \\
3 \mathrm{Mbps}\end{array}$ & $\begin{array}{r}\text { Very High } \\
7 \text { Gbps }\end{array}$ \\
\hline Coverage & Small & Medium & High & High & Very High & Very High & Small & Medium & Small \\
\hline Up to & $100 \mathrm{~m}$ & $1 \mathrm{~km}$ & $10 \mathrm{~km}$ & $50 \mathrm{~km}$ & $30 \mathrm{~km}$ & $30 \mathrm{~km}$ & $10 \mathrm{~m}$ & $100 \mathrm{~m}$ & $10 \mathrm{~m}$ \\
\hline V2I & Yes & Yes & Yes & Yes & Yes & Yes & Yes & Yes & Yes \\
\hline $\mathrm{V} 2 \mathrm{~V}$ & Yes & Yes & No & No & No & Partial(D2D) & Yes & Yes & Yes \\
\hline
\end{tabular}

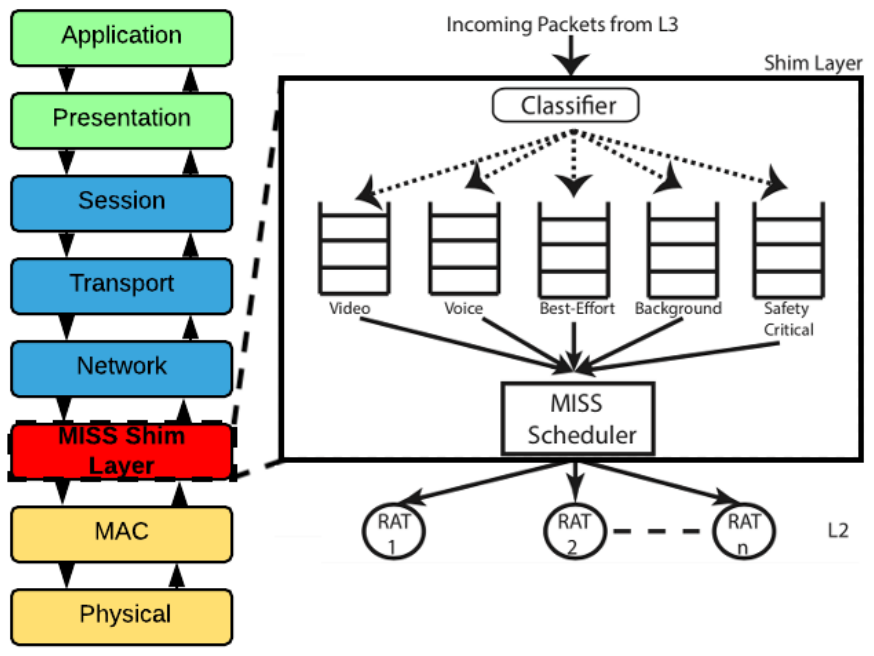

Fig. 2. Illustration of the Wi-Fi Standard with the MISS shim layer and details of the shim layer structure.

network and MAC layers was proposed [2]. The transparent implementation with the MAC and Physical standards and the details of the shim layer are shown in Fig. 2. The task of the shim layer, is to provide an independent layer over which network, transport and application layer protocols can function efficiently, independent of the access technologies used in each of the point-to-point links in an end-to-end connection.

The shim layer consists of a classifier, five traffic category queues and a Multiple Interface Scheduling System (MISS). The MISS system is divided in two asynchronous parts: the utility scoring system and the scheduler. The scoring system comprises different utility functions and reference values based on a weighted Multiple Attribute Decision Making (MADM). Six attributes (bandwidth, cost, energy consumption, delay, SINR, and speed of vehicle) are considered for the algorithm. The scheduler makes use of the scores provided by a scoring system to distribute the packets across different RATs, simultaneously at each of its iterations. After monitoring the queue sizes, the scheduler requests a score calculation and sends the packets to the appropriate RATs based on the received scores, making the process transparent for the upper layers. The full details have been described in [25]. It is to be noted that different types of algorithms (e.g. Game Theory) can be implemented to provide the scores for the MISS scheduler.

In the following sections, the performance of the shim layer scheduler approach will be compared to a standard Switched approach, where a node remains connected to an Access Point until it looses connection and then seeks an alternative.

\section{Simulation ENVIRONMENT}

The simulation aims to analyse the ability of the smart scheduler to prioritise packets based on QoS, in this case safety messages, in the presence of video traffic.

The simulation setup is listed in Table II. There are a varying number of nodes, 10 to 50 with increments of 10 for each iteration, with a random mobility model in a 62,500 $\mathrm{m}^{2}$ area. 20 nodes are depicted in red in Fig. 3. With the specified ranges and the simulated environment any node has access to at least two $802.11 \mathrm{p}$ side units. Since $802.11 \mathrm{p}$ is not infrastructure based, the nodes can communicate directly between them, in an ad-hoc mode. However, due to the setup of the simulation there are no safety messages exchanged directly between nodes as they all transmit information to the central control unit, also known as the receiver sink, seen in grey at coordinates $(0,10)$ in Fig. 3. All nodes act in a greedy manner and individually try to get as many packets through as possible. There is no cooperation between the users. All nodes are on the same channel and thus there is strong interference/collision.

The defined area assumes access points are located both indoor (802.11n) and outdoor (802.11p). Different propagation loss models are applied based on their assumed location. The default ns-3 'Log Distance Propagation Loss Model' with a path loss distance exponent of 3 is used for the 802.11n 2.4 
TABLE II

Multiple Users NS-3 Simulation Setup

\begin{tabular}{lrrrr}
\hline & Wi-Fi (1) & Wi-Fi (2) & Wi-Fi (3) $\bigcirc$ \\
RAT & $802.11 \mathrm{p}$ & $802.11 \mathrm{n}$ & $802.11 \mathrm{n}$ \\
Frequency & $5.9 \mathrm{GHz}$ & $2.4 \mathrm{GHz}$ & $5.18 \mathrm{GHz}$ \\
Data Rate & $6 \mathrm{Mbps}$ & $21.9 \mathrm{Mbps}$ & $27 \mathrm{Mbps}$ \\
Link Delay & $2.40 \mathrm{~ms}$ & $1.73 \mathrm{~ms}$ & $1.70 \mathrm{~ms}$ \\
APs & 12 & 16 & 9 \\
Propagation LM & Two-ray & Log Distance & Log Distance \\
Transmission Range & $125 \mathrm{~m}$ & $50 \mathrm{~m}$ & $50 \mathrm{~m}$ \\
\hline AP Spacing & $50 \mathrm{~m}$ & Safety Traffic & $2 \mathrm{kbps}$ \\
Transmit Power & $20 \mathrm{dBm}$ & Other Traffic & $30 \mathrm{Mbps}$ \\
Number of nodes & {$[10 ; 50]$} & Transport Layer & UDP \\
Node speed & $5 \mathrm{~m} / \mathrm{s}$ & Network Layer & IPv6 \\
Mobility & Random & Addressing & Static \\
Area & $62,500 \mathrm{~m}{ }^{2}$ & Receiver Sink & 1 \\
Queue Type & CoDel & Packet Size & 1448 bytes \\
& & Safety Packet Size & 200 bytes \\
Simulator & $\mathrm{ns}-3.22$ & Simulation Time & $100 \mathrm{~s}$ \\
\hline & & &
\end{tabular}

$\mathrm{GHz}$ and $5.18 \mathrm{GHz}$ as the existing Wi-Fi APs are usually indoors. The log-distance propagation loss model is a radio propagation model that predicts the path loss that a signal encounters inside a building or densely populated areas as a function of distance. In addition, the maximum transmission ranges have been set to $50 \mathrm{~m}$ and the AP spacing is $50 \mathrm{~m}$.

For the $802.11 \mathrm{p}$, it is assumed that the APs are placed in open space, also with a $50 \mathrm{~m}$ spacing. The propagation model is a 'Two-Ray Ground' loss model as these APs are assumed to be targeted towards vehicular communication, mounted on the road infrastructure and in line of sight with the connected mobile nodes. The maximum transmission range is $125 \mathrm{~m}$. For this simulation the safety tagged messages are set similar to the Basic Safety Messages (BSMs) [26]. BSM is a 200byte packet that is generally broadcast from every vehicle at a nominal rate of $10 \mathrm{~Hz}$.

A saturated environment is defined as one where the data rate required by the application layer can not be satisfied by a single RAT. In this scenario, the highest data rate RAT is Wi-Fi 802.11n 5.18 GHz with $27 \mathrm{Mbps}$. The simulation uses a combination of real-time video and BSM and investigates saturated and non saturated conditions. For the saturated condition the maximum video application data rate is $30 \mathrm{Mbps}$, which requires more than one RAT, and the BSM is $2 \mathrm{kbps}$. For the non-saturated condition, the video application data rate is 2 Mbps, while the BSM remains at $2 \mathrm{kbps}$. In the Switched case, one of the three RATs is accessed one at a time, following the current default Wi-Fi practice when a node only switches access point when it loses signal.

The simulation uses a 'Random Walk' mobility model. Although this model is not necessarily representative of the mobility of vehicles on roads, it does provide a more robust testing of the scheduler due to the non predictive movement,

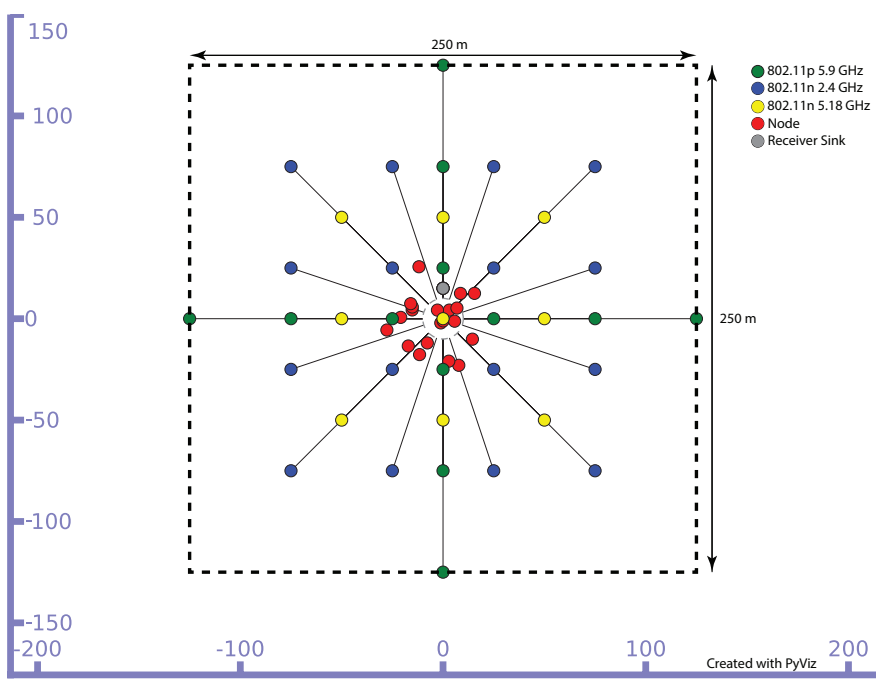

Fig. 3. Multi node simulation

and can represent the movement of other ITS nodes such as pedestrians and drones. This was done as an initial step to confirm the performance of the RAT switching behaviour. Having demonstrated this is a viable approach, the next step would be to repeat it in a more representative wireless environment. Several models have already been reported which can be used for this purpose [27].

\section{Results}

The results (Fig. 4) illustrate the performance of the prioritisation scheme for the BSM messages in the presence of video traffic. The results are given as average values for all users rather than individual nodes, and therefore indicates the overall benefit to the ITS user community.

Fig. 4(a) shows the results in the non saturated condition. It shows that by using the smart scheduler at the shim layer, the packet delay does not increase with the number of users, whereas with the Switched scheme (as defined in Section IV), the packet delay increases with the number of users and the average delay is significantly higher.

Fig. 4(b) shows the results in saturated conditions and indicated a similar performance to non-saturated conditions with the packet scheduler. The reason for the flattening in the 'Switched' approach above 40 users is that the number of packets received by the Sink remains constant due to saturation, as the calculation of the average only includes the successfully received packets.

Fig. 4(c) shows the Packet Delivery Ratio (PDF) results in non saturated conditions. For the 'Switched' approach, the PDR decreases as the numbers increase to a level of only $40 \%$ for 50 users, whereas with the packet scheduler, the PDR is maintained at $94 \%$ or above for up to 50 users.

Fig. 4(d) shows the results of PDR under saturated conditions. While the packet scheduler maintains a delivery level greater than $94 \%$ up to 50 users, whereas the switched 
approach the packet delivery ratio decrease less than $5 \%$ with 50 users.

As an overview, it can be observed that as the number of nodes increases, the shim layer Packet Delivery Ratio (PDR) and delay performs similarly in both saturated and nonsaturated conditions, while the switched scheme has lower performance as the number of nodes increases. This is the expected situation with Carrier Sense Multiple Access with Collision Avoidance (CSMA/CA) as the number of collisions increases with the number of users. The high number of users increases the back-off time which in return increases the delay of the packets. As this is expected for Wi-Fi $802.11 \mathrm{n} 2.4 \mathrm{GHz}$ and $5.18 \mathrm{GHz}, 802.11 \mathrm{p}$ should work even with a large number of nodes. It confirms the work by Han et al. [17] showing that IEEE $802.11 \mathrm{p}$ exhibits lower performance when there are a large number of vehicles.

Under certain conditions, safety packets have higher delays due to the random movement of the vehicles which can lead to a high density around certain APs, as can be seen in Fig. 3. There is a drop in the PDR with an increase of number of users, even with the packet scheduler approach. This is related to the fact that the safety messages in the simulation environment are not broadcasted to all the 802.11p APs, but only to the closest one to the node.

\section{FUTURE WORK}

Further work could include studying the impact of prioritisation on non-prioritised services. For instance, it could address the use of cooperative scheduling schemes, which may give performance improvements but may also lead to increased overhead. Processing groups of packets could be investigated, which may help to reduce overhead but may have an impact on the overall performance of a multi-user system. A study could also be carried out to compare the performance of UDP and TCP in a heterogeneous environment with a smart scheduler.

The increasing demand for higher bandwidth and lower delays is likely to lead to the introduction of new technologies such as millimeter-Wave (mmWave) and Visible Light Communication (VLC) in ITS.

mmWave devices offer several potential advantages for adhoc networks including reduced interference due to directional antennas and building blockages, as well as high bandwidth channels which can support large data rates. Thornburg et al. [28] show that mmWave networks support larger densities, higher area spectral efficiencies, and better rate coverage compared to traditional, lower-frequency ad-hoc networks. There are currently two standards that have emerged: IEEE 802.15.3c and IEEE 802.11ad.

The increasing interest in the VLC technology has led to the development of the IEEE 802.15.7 standard. Although it is part of the IEEE 802.15 standard, dedicated to personal area networks, the specifications explicitly consider vehicles and illuminated roadside devices, such as traffic lights or street lights among the addressed applications [29]. The key feature of VLC in relation to other vehicular RATs (DSRC, cellular) is that it operates in unlicensed and uncongested bands. Also, it

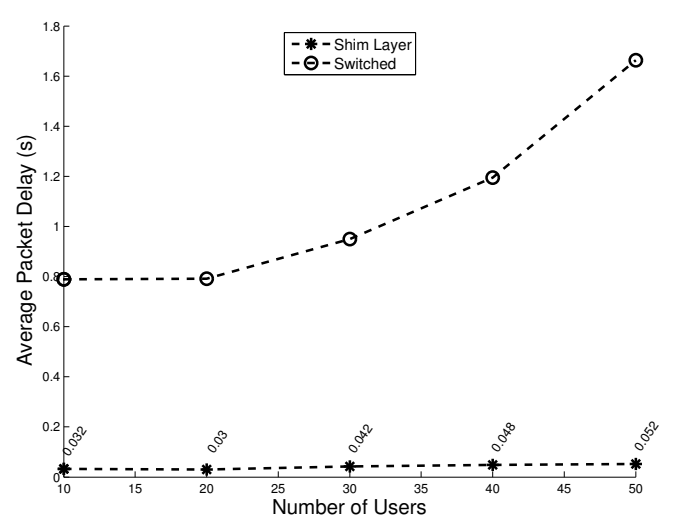

(a) Safety Messages Average Delay in a Non-Saturated Condition

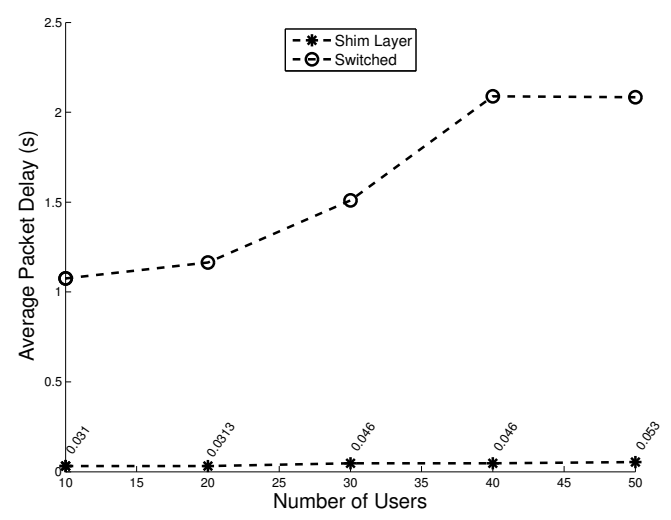

(b) Safety Messages Average Delay in a Saturated Condition

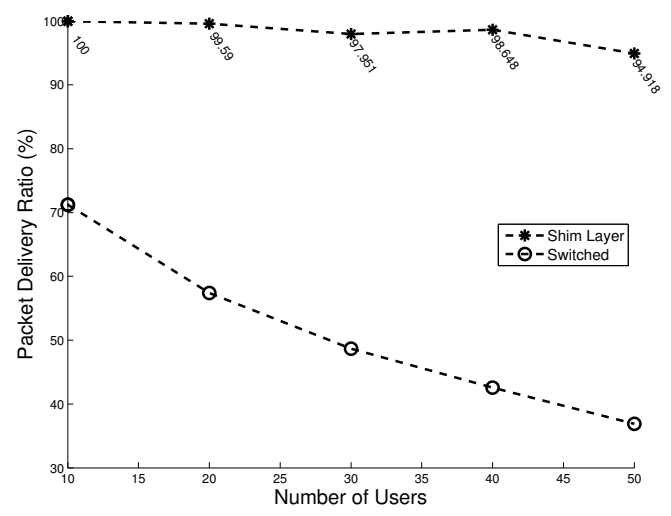

(c) Safety Messages Packet Delivery Ratio in a NonSaturated Condition

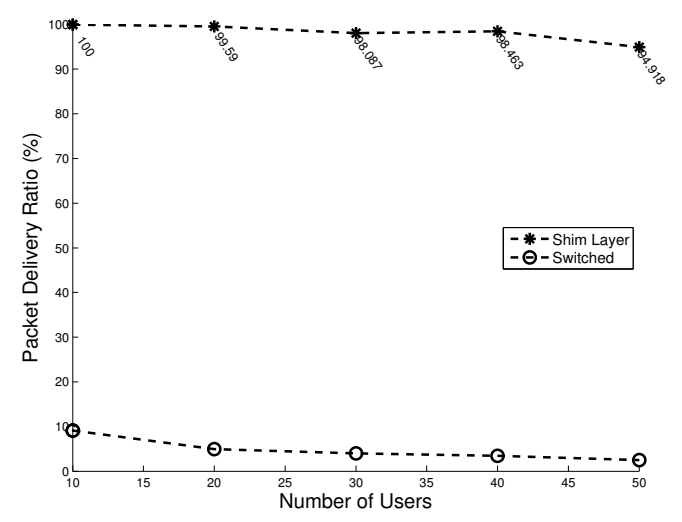

(d) Safety Messages Packet Delivery Ratio in a Saturated Condition

Fig. 4. Simulation Results 
can use the LEDs available on a vehicle as transmitters and the available infrastructure (street lights) as the access network.

It will be necessary to study how to incorporate these new RATs in heterogeneous systems with smart schedulers.

\section{CONCLUSION}

This paper considers the use of heterogeneous wireless techniques to support multiservice provision in a non-cooperative multi-user ITS environment. The approach uses a packet scheduler to respond to QoS requirements of applications. Performance is optimised by scheduling packets to the best available radio access technology. Packet scheduling is determined at the user node and takes place at a shim layer below the network layer which allows each user to be transparent to any available wireless technology. The paper presents results of simulation experiments to analyse the performance of this scheme in a non-cooperative multi node environment. The results show that by using a packet scheduler in a shim layer approach, the delay and packet delivery ratios are significantly lower than those achieved using a switched approach, in both non-saturated and saturated conditions.

\section{REFERENCES}

[1] E. Gustafsson and A. Jonsson, "Always best connected," Wireless Commun., vol. 10, no. 1, pp. 49-55, Feb. 2003. [Online]. Available: http://dx.doi.org/10.1109/MWC.2003.1182111

[2] C. Roman, P. Ball, and S. Ou, "A shim layer for heterogeneous wireless communications scheduling in intelligent transport systems," in 2015 IEEE Symposium on Computers and Communication (ISCC), July 2015, pp. 174-179. [Online]. Available: https://dx.doi.org/10.1109/ ISCC.2015.7405512

[3] — , "Multiple interface scheduling system for heterogeneouswireless vehicular networks: Description and evaluation." EAI Endorsed Trans. Wireless Spectrum, vol. 3, no. 11, 2017.

[4] M. Kihl, K. Br, P. Mahanta, and E. Coelingh, "3gpp lte downlink scheduling strategies in vehicle-to-infrastructure communications for traffic safety applications," in Computers and Communications (ISCC), 2012 IEEE Symposium on, July 2012, pp. 000448-000 453.

[5] E. Hossain, G. Chow, V. C. M. Leung, R. D. McLeod, J. Mišić, V. W. S. Wong, and O. Yang, "Vehicular telematics over heterogeneous wireless networks: A survey," Comput. Commun., vol. 33, no. 7, pp. 775-793, may 2010. [Online]. Available: http://dx.doi.org/10.1016/j. comcom.2009.12.010

[6] I. Lequerica, P. M. Ruiz, and V. Cabrera, "Improvement of vehicular communications by using $3 \mathrm{~g}$ capabilities to disseminate control information," IEEE Network, vol. 24, no. 1, pp. 32-38, Jan 2010. [Online]. Available: http://dx.doi.org/10.1109/MNET.2010.5395781

[7] H. T. Cheng, H. Shan, and W. Zhuang, "Infotainment and road safety service support in vehicular networking: From a communication perspective," Mechanical Systems and Signal Processing, vol. 25, no. 6 , pp. 2020-2038, 2011. [Online]. Available: http://dx.doi.org/10.1016/j. ymssp.2010.11.009

[8] Z. Hameed Mir and F. Filali, "Lte and ieee $802.11 \mathrm{p}$ for vehicular networking: a performance evaluation," EURASIP Journal on Wireless Communications and Networking, vol. 2014, no. 1, pp. 1-15, 2014. [Online]. Available: http://dx.doi.org/10.1186/1687-1499-2014-89

[9] K. Zheng, L. Zhang, W. Xiang, and W. Wang, Heterogeneous Vehicular Networks, ser. SpringerBriefs in Electrical and Computer Engineering. Springer International Publishing, 2016. [Online]. Available: https://books.google.co.uk/books?id=OATNCwAAQBAJ

[10] G. Araniti, C. Campolo, M. Condoluci, A. Iera, and A. Molinaro, "Lte for vehicular networking: a survey," IEEE Communications Magazine, vol. 51, no. 5, pp. 148-157, May 2013. [Online]. Available: http://dx.doi.org/10.1109/MCOM.2013.6515060

[11] H. Karimi, G. Koudouridis, and K. Dimou, "On the spectral efficiency gains of switched multi-radio transmission diversity," Proceedings of WPMC 05, Aalborg, Danmark, 2005.
[12] G. P. Koudouridis, P. Soldati, H. Lundqvist, and C. Qvarfordt, "Usercentric scheduled ultra-dense radio access networks," in $201623 \mathrm{rd}$ International Conference on Telecommunications (ICT), May 2016, pp. $1-7$.

[13] M. K. Jiau, S. C. Huang, J. N. Hwang, and A. V. Vasilakos, "Multimedia services in cloud-based vehicular networks," IEEE Intelligent Transportation Systems Magazine, vol. 7, no. 3, pp. 62-79, Fall 2015.

[14] Y. He, M. Chen, B. Ge, and M. Guizani, "On wifi offloading in heterogeneous networks: Various incentives and trade-off strategies," IEEE Communications Surveys Tutorials, vol. PP, no. 99, pp. 1-1, 2016.

[15] M. Picone, S. Busanelli, M. Amoretti, F. Zanichelli, and G. Ferrari, Advanced technologies for intelligent transportation systems. Springer, 2015, vol. 139

[16] V. Bychkovsky, B. Hull, A. Miu, H. Balakrishnan, and S. Madden, "A measurement study of vehicular internet access using in situ wi-fi networks," in Proceedings of the 12th Annual International Conference on Mobile Computing and Networking, ser. MobiCom '06. New York, NY, USA: ACM, 2006, pp. 50-61. [Online]. Available: http://doi.acm.org/10.1145/1161089.1161097

[17] C. Han, M. Dianati, R. Tafazolli, R. Kernchen, and X. Shen, "Analytical study of the ieee 802.11 p mac sublayer in vehicular networks," IEEE Transactions on Intelligent Transportation Systems, vol. 13, no. 2, pp. 873-886, June 2012

[18] A. Bazzi, "On uncoordinated multi user multi rat combining," in Vehicular Technology Conference (VTC Fall), 2011 IEEE, Sept 2011, pp. 1-6. [Online]. Available: https://dx.doi.org/10.1109/VETECF.2011. 6093056

[19] A. K. L. Miu, "Improving packet delivery efficiency using multi-radio diversity in wireless lans," Ph.D. dissertation, Massachusetts Institute of Technology, 2006

[20] G. P. Koudouridis, A. Yaver, and M. U. Khattak, "Performance evaluation of multi-radio transmission diversity for tcp flows," in Vehicular Technology Conference, 2009. VTC Spring 2009. IEEE 69th, April 2009, pp. 1-5.

[21] A. L. Ramaboli, O. E. Falowo, and A. H. Chan, "Bandwidth aggregation in heterogeneous wireless networks: A survey of current approaches and issues," Journal of Network and Computer Applications, vol. 35, no. 6, pp. 1674 - 1690, 2012. [Online]. Available: http://dx.doi.org/10.1016/j.jnca.2012.05.015

[22] K. Habak, K. A. Harras, and M. Youssef, "Bandwidth aggregation techniques in heterogeneous multi-homed devices: A survey," Computer Networks, vol. 92, Part 1, pp. 168 - 188, 2015. [Online]. Available: http://www.sciencedirect.com/science/article/pii/S1389128615002996

[23] D. Kaspar, K. Evensen, P. Engelstad, A. F. Hansen, P. 1. Halvorsen, and C. Griwodz, "Enhancing video-on-demand playout over multiple heterogeneous access networks," in 2010 7th IEEE Consumer Communications and Networking Conference, Jan 2010, pp. 1-5. [Online]. Available: https://dx.doi.org/10.1109/CCNC.2010.5421846

[24] A. Yaver and G. P. Koudouridis, "Performance evaluation of multi-radio transmission diversity: Qos support for delay sensitive services," in Vehicular Technology Conference, 2009. VTC Spring 2009. IEEE 69th, April 2009, pp. 1-5. [Online]. Available: https: //dx.doi.org/10.1109/VETECS.2009.5073791

[25] C. Roman, P. Ball, and S. Ou, "Performance evaluation of dynamic scheduler in multiple queue shim layer for heterogeneous vehicular communications," in 2015 IEEE Globecom Workshops (GC Wkshps), Dec 2015, pp. 1-6. [Online]. Available: https: //dx.doi.org/10.1109/GLOCOMW.2015.7414037

[26] "Ieee standard for wireless access in vehicular environments (wave) - networking services," IEEE Std 1609.3-2016 (Revision of IEEE Std 1609.3-2010), pp. 1-160, April 2016.

[27] M. Boban and T. Vinhoza, "Modeling and simulation of vehicular networks: towards realistic and efficient models," in Mobile Ad-Hoc Networks, X. Wang, Ed. Rijeka: InTech, 2011, ch. 3. [Online]. Available: https://doi.org/10.5772/12846

[28] A. Thornburg, T. Bai, and R. W. Heath, "Mmwave ad hoc network coverage and capacity," in 2015 IEEE International Conference on Communications (ICC), June 2015, pp. 1310-1315.

[29] A. Bazzi, B. M. Masini, A. Zanella, and A. Calisti, "Visible light communications as a complementary technology for the internet of vehicles," Computer Communications, pp. -, 2016. [Online]. Available: http://www.sciencedirect.com/science/article/pii/S0140366416302626 